\title{
Elevated SARS-Cov-2-Specific IgM Levels Indicate Clinically Unfavorable Outcomes in Patients with COVID-19: A Retrospective Cohort Study
}

\author{
Zhiquan $\mathrm{Hu}^{\mathrm{l}} *$ * \\ Jingjing $\mathrm{Li}^{2, *}$ \\ Chunguang Yang (D) \\ Fen $\mathrm{Chen}^{3}$ \\ Zhihua Wang \\ Ping Lei ${ }^{4}$ \\ Guanxin Shen ${ }^{4}$ \\ 'Department of Urology, Tongji Hospital, \\ Tongji Medical College, Huazhong \\ University of Science and Technology, \\ Wuhan, People's Republic of China; \\ ${ }^{2}$ Department of Clinical Laboratory, \\ Union Hospital, Tongji Medical College, \\ Huazhong University of Science and \\ Technology, Wuhan, People's Republic of \\ China; ${ }^{3}$ Department of Cardiology, Union \\ Hospital, Tongji Medical College, \\ Huazhong University of Science and \\ Technology, Wuhan, People's Republic of \\ China; ${ }^{4}$ Department of Immunology, \\ Tongji Medical College, Huazhong \\ University of Science and Technology, \\ Wuhan, People's Republic of China \\ *These authors contributed equally to \\ this work
}

Correspondence: Chunguang Yang Department of Urology, Tongji Hospital, Tongii Medical College, Huazhong

University of Science and Technology,

No. 1095, Jiefang Road, Wuhan, 430030,

People's Republic of China

Tel +86- | 387| 084024

Fax +86-27-83663460

Email cgyang-hust@hotmail.com

Fen Chen

Department of Cardiology, Union

Hospital, Tongji Medical College,

Huazhong University of Science and

Technology, Wuhan, People's Republic of

China

Email 54II3447@qq.com
Background: COVID-19 outbreak began in Wuhan and pandemics occur. Although SARSCoV-2-specific immunoglobulins have been detected in serum of COVID-19 patients, their dynamics and association with outcomes have not been fully characterized.

Methods: This retrospective cohort study investigated the association between SARS-CoV -2-specific immunoglobulins and clinical outcomes of COVID-19 patients. We recruited 137 participants who were diagnosed with COVID-19 in four wards of the Tongji Hospital in Wuhan, China. Among the 137 participants, 81 patients were recovered, 23 patients died, and 33 patients remained hospitalized by the end of the study. SARS-CoV-2-specific immunoglobulins were analyzed by chemiluminescence assays. Laboratory and radiological characteristics, and clinical outcomes were compared between the recovered group and the deceased group. Furthermore, a matched cohort study was conducted in which each nonsurvivor was matched to two recovered patients of similar age.

Results: SARS-CoV-2-specific IgM levels peaked in the fourth week after the onset of COVID-19, while serum IgG levels rose earlier and remained high up to the eighth week. In the age-matched cohort study, the serum IgM, but not IgG levels, were higher among the non-survivors than in the recovered group $(\mathrm{P}=0.006)$. The area under the ROC curve for the IgM and IgG levels was 0.702 (95\% CI: $0.560-0.845, \mathrm{P}=0.006)$ and $0.596(95 \%$ confidence interval: $0.449-0.744, \mathrm{P}=0.194)$, respectively. We also showed that patients with COVID-19 who had high IgM or IgG levels (stratified according to best cut-off) exhibited significantly lower overall survival (Kaplan-Meier survival curves, $\mathrm{P}<0.05$ ).

Discussion: These results indicate the association between immunoglobulins and outcome in patients with COVID-19 and demonstrated that elevated serum IgM levels could indicate poor outcomes in patients with COVID-19. Further, the information about the profile of SARS-CoV-2-specific IgGs may be useful for the future epidemiological investigations of COVID-19 therapies.

Keywords: SARS-CoV-2, COVID-19, immunoglobulin, prognosis, antibodies, IgM, IgG, diagnosis

\section{Introduction}

Coronavirus disease 2019 (COVID-19) has spread rapidly throughout the world since its discovery in December 2019. ${ }^{1,2}$ As of October 16, 2020, more than $1,095,000$ deaths attributable to the severe acute respiratory syndrome coronavirus 2 (SARS-CoV-2) infection have been reported. ${ }^{3}$ While effective strategies are urgently needed for an early control of the disease, to date, only a few drugs have shown efficacy for the treatment of COVID-19. ${ }^{4}$ 
The pathogenesis and pathophysiology for COVID-19 are still unclear. Immune function may play a vital role in SARS-CoV-2 infection. Several researches have shown changes of cytokines, lymphocyte subsets, and the dysregulation of host immune response for patients with different severities of COVID-19. Antibodies are the key components in the immune response to viral infections ${ }^{5,6}$ and serology testing has important implications for epidemiological assessments, serological therapies, vaccine development, and the possible use of what has been termed "immunity certificates". 7 Previous studies have reported detections of elevated levels of SARS-CoV -2-specific immunoglobulin M (IgM) and immunoglobulin $\mathrm{G}$ (IgG) in the serum of patients with COVID-19. ${ }^{8}$ Although, immunological studies have indicated simultaneous or earlier IgG than IgM seroconversion in most cases, ${ }^{9,10}$ scant information is available about the profiles of $\operatorname{IgG}$ and IgM and their associations with the outcomes in patients with COVID-19. Therefore, the present study aimed to examine the relationship between IgG and IgM levels as an indicator of the clinical outcomes of patients with COVID-19.

\section{Materials and Methods}

\section{Study Design and Participants}

A total of 104 hospitalised COVID-19 patients with definitive clinical outcome and detectable levels of serum IgM and IgG against SARS-CoV-2 examined between
February 23, 2020, and March 23, 2020 in four wards of Tongji Hospital were retrospectively investigated. All adult patients who were diagnosed with COVID-19 in four wards of the Tongji Hospital ("The specific hospital for the treatment of severe patients with COVID-19 in Wuhan", as designated by the Chinese government). Diagnoses were confirmed using SARS-CoV-2 real-time RT-PCR or serum IgM and IgG antibody testing according to the Seventh Revised Trial Version of the Novel Coronavirus Pneumonia Diagnosis and Treatment Guidance of China (available from http://www.nhc.gov.cn/yzygj/s7653p/ 202003/46c9294a7dfe4cef80dc7f5912eb1989.shtml). Data were collected between February 23, 2020, and March 23, 2020. Patients diagnosed with COVID-19 were enrolled with detected levels of serum IgM and IgG against SARSCoV-2. This study was reviewed and approved by the Medical Ethics Committee of the Tongji Hospital of Huazhong University of Science and Technology (IRB ID: TJ- IRB20200343), and was exempted from the need to obtain informed consent due to the particular circumstances of the disease outbreak. Information was collected from the hospital's electronic medical record system. Clinical, laboratory, and radiological parameters, as well as clinical outcomes were obtained using standardized forms completed by all participants involved in the study. Two researchers independently reviewed the data. The inclusion and exclusion criteria for the participants of the study are summarized in Figure 1.

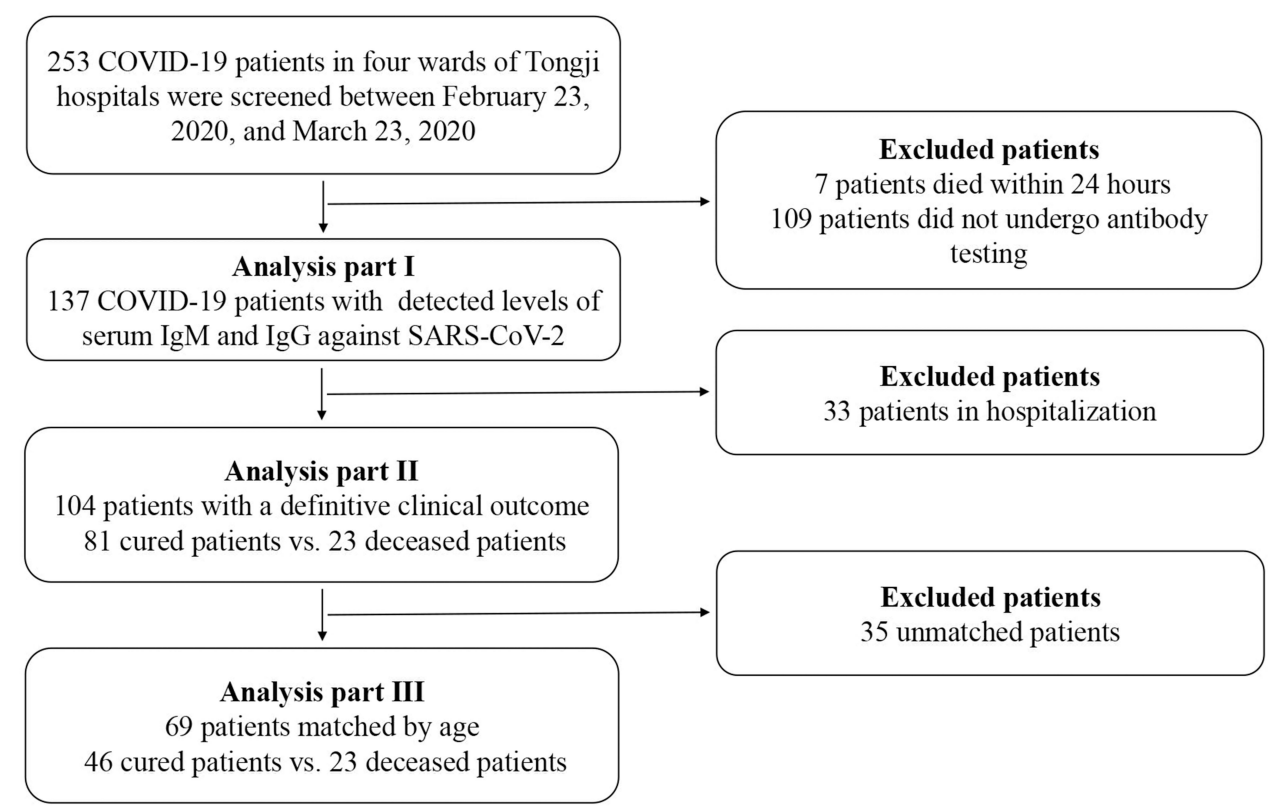

Figure I Study profile (inclusion and exclusion criteria). 


\section{Antibody Assay}

Serum IgM and IgG antibodies against SARS-CoV-2 spike protein $\mathrm{S}$ and nucleocapsid protein $\mathrm{N}$ were detected by a chemiluminescence kit (iFlash-SARS-CoV-2 IgM, iFlash-SARS-CoV-2 IgG) and an iFlash 3000 CLIA System supplied by Yhlo Biotech Co., Ltd. (Shenzhen, China). The chemiluminescence kit was certified by the EU CE. A threshold of $10 \mathrm{AU} / \mathrm{mL}$ was used for both IgM and $\mathrm{IgG}$, as recommended by the manufacturer.

In order to quantify $\mathrm{T}$ lymphocyte subsets, $1 \times 106$ PBMCs were stained with indicated antibodies (CD3APC, CD4-PerCP, and CD8-FITC antibodies all from BD Biosciences) in the dark at room temperature for $20 \mathrm{~min}$. After two washes, the cells were analyzed within $1 \mathrm{~h}$. All samples were detected by BD FACS Canto II Flow Cytometry System and analyzed with the BD FACS Diva Software. In order to explore the influence of COVID-19 on the secretion of cytokines and receptors, chemiluminescence immunoassay (CLIA) was performed. Cytokines and receptors including interleukin 2 receptor (IL-2R), IL6 , IL-8, IL-10 and TNF- $\alpha$ were assessed using serum samples (Immulite 1000, DiaSorin Liaison, Italy; or Cobas e602, Roche Diagnostics, Germany). The testing kit was purchased from Roche Diagnostics.

\section{Statistical Analysis}

The statistical software SPSS 23.0 (SPSS Inc., Chicago, IL, USA) was used to analyze the data in this study. Categorical variables are described as frequencies and percentages, and continuous variables are described using the means or medians with interquartile ranges (IQRs). Normally distributed continuous variables were analyzed using Student's $t$-test and non-normal data was analyzed using Mann-Whitney test. Proportions of categorical variables were compared using the $\chi^{2}$ test. Receiver operating characteristic (ROC) curves were used to evaluate $\operatorname{IgM}$ and IgG as potential predictors of the clinical outcomes. Pearson correlation analysis was performed to assess association of IgM levels with clinical outcomes. All tests were 2 -sided, and results with $\mathrm{P}<0.05$ were considered statistically significant.

\section{Results}

\section{Patient Characterization}

The participants in this study comprised of 82 males and 55 females. Among the 137 participants, 81 patients were recovered, 23 patients died, and 33 patients remained hospitalized during the study period. Results from the antibody assays showed that SARS-CoV-2-specific IgM levels peaked during the fourth week following the onset of COVID-19 in the patients, while serum IgG levels rose quickly during the first two weeks following onset and remained high until the eighth week (Figure 2A). Moreover, seroconversion of $\mathrm{IgG}$ occurred simultaneous with or earlier than that of IgM during the early stages. Of the 104 patients in the recovered and deceased group, 66 (63\%) had one or more comorbidities (Table 1); hypertension (39 [38\%]), diabetes (21 [20\%]), cardiovascular disease (11 [11\%]), COPD (2 [2\%]), malignancy $(8[8 \%])$, and chronic kidney disease $(4[4 \%])$ were the most common coexisting conditions. The chief complaints were fever $(83[80 \%])$, cough $(15[14 \%])$, and other uncommon symptoms (Table 1). The results also showed that $97(93 \%)$ of the 104 patients presented bilateral lung involvement, as was shown by chest $\mathrm{CT}$ or X-ray images. The mean hospitalization duration was $22.8(12.0-30.0)$ days. The patients who died were significantly older vs recovered (70.1 years [IQR 65.0-79.0] vs 59.5 years [IQR 50.0-69.0]; $\mathrm{P}=0.001)$ ) and had a higher likelihood of comorbidities (22 [96\%] vs 44 [54\%]; P < 0.001).

\section{Laboratory Parameters in Recovered Patients and Non-Survivors}

The blood counts of patients at the time of admission showed significant reductions in the lymphocyte counts; this was especially pronounced in the patients who died later (Table 2). Consistent with this observation, most subset counts of lymphocytes ( $\mathrm{T}$ cells, Th cells, Ts cells, and NK cells) were significantly higher in the recovered patients than in non-survivors. The levels of all cytokines detected (IL2R, IL6, IL8, IL10, and TNF- $\alpha$ ) were significantly higher in non-survivors than in survivors. No significant difference was observed in the B cell counts between the non-survivors $(143.4 / \mu \mathrm{L}[33.0-225.0])$ and the recovered patients $(166.1 / \mu \mathrm{L}$ [84.5-200.8], $\mathrm{P}=0.569)$.

\section{Association of IgM Levels with Clinical Outcomes}

Serum IgM levels were higher in the non-survivors (IgM 231.6 AU/mL [30.4-296.2]) than in the recovered patients ( $\operatorname{IgM} 84.8$ $\mathrm{AU} / \mathrm{mL}$ [12.4-74.4], $\mathrm{P}=0.006$ ); however, this was not observed for IgG (234.8 AU/mL [IQR 157.3-295.5] in nonsurvivors vs 195.0 AU/mL [IQR 149.5-230.1] in recovered patients; $\mathrm{P}=0.061$; Table 2, Figure $2 \mathrm{~B}$ and $\mathrm{C}$ ). The correlation analysis showed that serum IgM levels negatively correlated with clinical outcomes $(r=-0.270, P=0.006)$. Further 

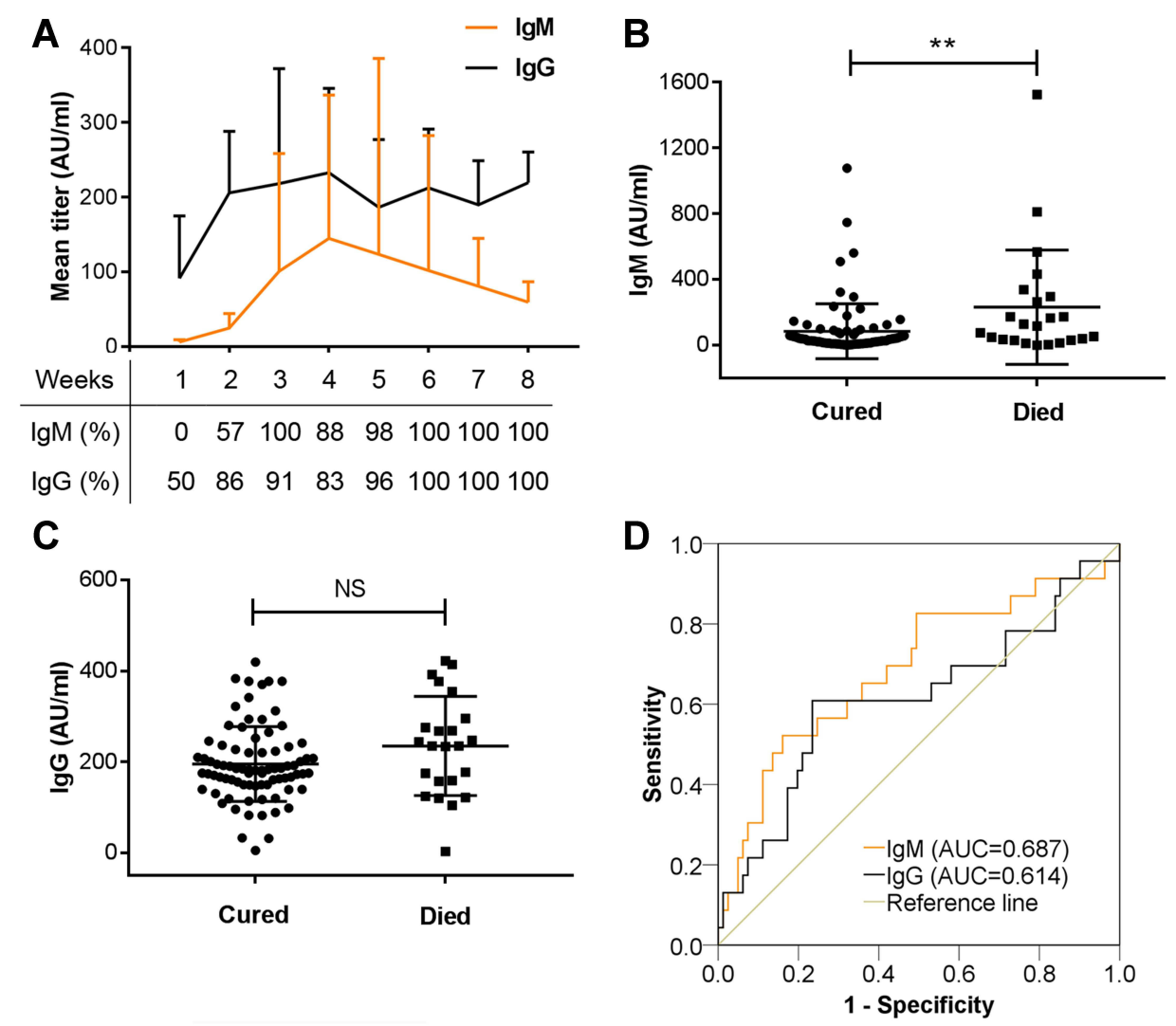

Figure 2 Serum SARS-Cov-2-specific IgM and IgG antibodies in patients with COVID-19. (A) Changes in serum SARS-Cov-2-specific IgM and IgG titers and positive rates over time ( $\mathrm{n}=137$ participants). (B) Serum IgM levels were significantly higher in the deceased group than in the recovered group. **P < $00 \mathrm{I}$. (C) No difference was found in the IgG levels between the recovered group and the deceased group. (D) Accuracy of serum IgM levels and IgG levels in predicting the clinical outcomes of COVID-I9 patients.

Abbreviation: NS, non-significant.

assessments of the predictive ability of the IgM and IgG levels with regard to the clinical outcomes were evaluated by ROC curve analysis. The area under the ROC curve (AUC) for IgM was determined to be 0.687 (95\% confidence interval: $0.553-$ $0.821, \mathrm{P}=0.006)$, whereas the AUC for IgG was $0.614(95 \%$ confidence interval: $0.469-0.759, \mathrm{P}=0.096$ ) (Figure 2D). There were no significant differences in the time interval between the disease onset and antibody detection between the non-survivors (29.7 days [24.0-35.0]) and the recovered patients (32.3 days [29.0-39.5], $\mathrm{P}=0.232$ ) (Table 2). Furthermore, we found that the IgM level was positively correlated with the duration of positivity for viral RNA $(\mathrm{P}<0.05)$.

\section{Cohort Study Matching by Age}

To minimize the confounding effects of age, a matched cohort study was performed. Each deceased patient was matched with two recovered patients of similar age (difference within four years). Consistent with the initial analysis, the leucocyte and neutrophil counts of patients were significantly higher in the deceased group than in the recovered group, whereas the lymphocyte subset count and eosinophil count were significantly lower in the deceased group than in the recovered group (Table 3). As in the prior analysis, the levels of all cytokines detected (IL2R, IL6, IL8, IL10, and TNF- $\alpha$ ) were significantly higher in non-survivors than in survivors $(\mathrm{P}<0.05)$.

The serum IgM level was higher in the non-survivors (IgM 231.6 AU/mL [30.4-296.2]) than in the recovered patients (IgM 72.3 AU/mL [13.0-56.2], P = 0.006); however, the IgG level was not (Table 3; Figure $3 \mathrm{~A}$ and $\mathrm{B}$ ). Moreover, the serum IgM levels negatively correlated with the clinical outcomes $(\mathrm{r}=-0.315, \mathrm{P}=0.008)$. The results from the ROC curve analysis (used to determine the ability of the $\operatorname{IgM}$ and $\operatorname{IgG}$ levels to predict the clinical outcomes) showed that the AUC for the IgM level was 0.702 (95\% confidence interval: $0.560-0.845, \mathrm{P}=0.006$ ), whereas the AUC for the IgG level was 0.596 (95\% confidence interval: $0.449-0.744, \mathrm{P}=0.194$ ) (Figure $3 \mathrm{C}$ ). As shown by Kaplan-Meier survival curves, COVID-19 patients with 
Table I Baseline Characteristics of COVID-19 Patients with Definitive Clinical Outcomes

\begin{tabular}{|c|c|c|c|c|}
\hline & Total $(n=104)$ & Recovered $(n=8 I)$ & Deceased $(n=23)$ & $P$ value \\
\hline Age, years & $62(51.0-71.0)$ & $59.5(50.0-69.0)$ & $70.1(65.0-79.0)$ & 0.001 \\
\hline Sex & & & & 0.113 \\
\hline Male & $62(60)$ & $45(56)$ & $17(74)$ & \\
\hline Female & $42(40)$ & $36(44)$ & $6(26)$ & \\
\hline Comorbidities & $66(63)$ & $44(54)$ & $22(96)$ & $<0.001$ \\
\hline Hypertension & $39(38)$ & $29(36)$ & $10(43)$ & 0.502 \\
\hline Diabetes & $2 I(20)$ & $17(21)$ & $4(17)$ & 0.705 \\
\hline Cardiovascular disease & $\mathrm{II}(\mathrm{II})$ & $6(7)$ & $5(22)$ & 0.049 \\
\hline COPD & 2(2) & $\mathrm{I}(\mathrm{I})$ & $\mathrm{I}(4)$ & 0.337 \\
\hline Malignancy & $8(8)$ & $3(4)$ & $5(22)$ & 0.004 \\
\hline Chronic kidney disease & $4(4)$ & $3(4)$ & $\mathrm{I}(4)$ & 0.887 \\
\hline Others & $17(16)$ & $9(I I)$ & $8(35)$ & 0.007 \\
\hline \multicolumn{5}{|l|}{ Chief complaint } \\
\hline Fever & $83(80)$ & $65(80)$ & $18(78)$ & 0.834 \\
\hline Cough & $15(14)$ & $10(12)$ & $5(22)$ & 0.258 \\
\hline Dyspnoea & $2(2)$ & $2(2)$ & $0(0)$ & 0.447 \\
\hline Others & $4(4)$ & $4(5)$ & $0(0)$ & 0.277 \\
\hline SARS-CoV-2 RNA & & & & 0.207 \\
\hline Positive & $86(83)$ & $69(85)$ & $17(74)$ & \\
\hline Negative & $18(17)$ & $12(15)$ & $6(26)$ & \\
\hline Bilateral involvement & & & & 0.605 \\
\hline Yes & $97(93)$ & $75(93)$ & $22(96)$ & \\
\hline No & 7) & $6(7)$ & $\mathrm{I}(4)$ & \\
\hline Hospital stay, days & $22.8(12.0-30.0)$ & $22.2(11.0-30.0)$ & $24.9(21.0-32.0)$ & 0.266 \\
\hline
\end{tabular}

Abbreviation: COPD, chronic obstructive pulmonary disease.

high IgM or IgG (stratified according to best cut-off) exhibited significantly lower overall survival $(\mathrm{P}<0.05)$ (Figure 3D and E).

\section{Discussion}

In this retrospective study, we analyzed profiles of the SARS-CoV-2-specific IgM and IgG antibodies among 137 hospitalized patients with confirmed COVID-19 diagnoses. We analyzed the clinical parameters of 104 hospitalized patients with definitive clinical outcomes (combined recovered and deceased). The results showed that the distribution profiles of SARS-CoV-2-specific antibodies were similar to those against SARS-CoV-1. ${ }^{11}$ Serum IgM levels were relatively higher in the deceased group, were negatively correlated with the clinical outcomes, and could be used to predict mortality in patients with COVID-19, thereby identifying SARS-CoV-2 IgM as a stable prognostic marker for COVID-19.

SARS-CoV-2 is a coronavirus with a higher transmissibility and weaker pathogenicity than SARS-CoV-1. ${ }^{6,12}$ Most infected patients present mild symptoms, however, some die of fatal pneumonia. While the clinical characteristics, risk 
Table 2 Laboratory Findings of COVID-19 Patients with Definitive Clinical Outcomes

\begin{tabular}{|c|c|c|c|c|}
\hline & Total $(n=104 *)$ & Recovered $(n=8 \mid *)$ & Deceased $(n=23 *)$ & $P$ value \\
\hline Hemoglobin, g/L & |27.|(||7.0-138.0) & $126.9(\mid 17.0-137.5)$ & $128.2(|| 4.0-|4| .0)$ & 0.353 \\
\hline Alanine aminotransferase, U/L & $29.0(16.0-36.8)$ & $28.8(16.0-36.0)$ & $30.0(18.0-39.0)$ & 0.763 \\
\hline Aspartate aminotransferase, U/L & $31.4(20.0-37.3)$ & $29.4(19.5-33.5)$ & $38.3(24.0-53.0)$ & 0.025 \\
\hline Albumin, $g / L$ & $35.7(31.5-40.4)$ & $36.8(32.7-41.5)$ & $31.7(27.9-35.0)$ & 0.001 \\
\hline Total bilirubin, $\mu \mathrm{mol} / \mathrm{L}$ & II.4(6.4-14.6) & $10.7(6.1-\mid 4.6)$ & $14.0(9.2-17.3)$ & 0.040 \\
\hline Lactate dehydrogenase, U/L & $320.0(201.3-400.0)$ & $291.9(193.0-315.5)$ & $419.0(273.0-524.0)$ & 0.006 \\
\hline Blood urea nitrogen, $\mathrm{mmol} / \mathrm{L}$ & $7.0(3.9-7.5)$ & $5.5(3.8-6.5)$ & $12.6(6.0-11.6)$ & $<0.001$ \\
\hline Creatinine, $\mu \mathrm{mol} / \mathrm{L}$ & $96.3(61.0-92.8)$ & $82.1(58.5-82.5)$ & $\mid 46.4(74.0-\mid 22.0)$ & $<0.001$ \\
\hline Prothrombin time, seconds & $14.4(\mid 3.3-14.6)$ & |3.8(|3.2-14.4) & $16.5(14.6-16.6)$ & $<0.001$ \\
\hline D-dimer, $\mu g / m L$ & $3.3(0.3-2.6)$ & $2.2(0.3-1.9)$ & $6.9(0.8-17.7)$ & 0.001 \\
\hline $\lg M, A U / m L$ & $117.3(13.3-104.2)$ & $84.8(\mid 2.4-74.4)$ & $231.6(30.4-296.2)$ & 0.006 \\
\hline $\operatorname{lgG}, \mathrm{AU} / \mathrm{mL}$ & $203.8(149.5-246.6)$ & $195.0(\mid 49.5-230.1)$ & $234.8(157.3-295.5)$ & 0.061 \\
\hline Antibody detection after onset, days & $31.7(28.0-38.0)$ & $32.3(29.0-39.5)$ & $29.7(24.0-35.0)$ & 0.232 \\
\hline $\mathrm{IL} 2 \mathrm{R}, \mathrm{U} / \mathrm{mL}$ & $825.9(359.3-1105.5)$ & $707.8(300.5-9 \mid 8.8)$ & $1223.2(580.0-1780.8)$ & 0.001 \\
\hline IL6, pg/mL & $68.7(2.4-40.1)$ & $23.7(1.7-17.5)$ & $213.5(18.8-136.4)$ & $<0.001$ \\
\hline IL8, pg/mL & $45.7(9.3-34.0)$ & $30.5(7.9-24.2)$ & $96.8(13.3-84.0)$ & 0.004 \\
\hline ILI0, pg/mL & $4.8(0.0-7.1)$ & $3.8(0.0-6.0)$ & $8.3(0.0-11.4)$ & 0.044 \\
\hline TNF- $\alpha, p g / m L$ & $9.7(6.0-11.7)$ & $8.4(5.7-10.7)$ & $\mid 4.0(8.3-15.5)$ & 0.004 \\
\hline Leucocytes, $\times 10^{\wedge} 9 /$ per $L$ & 7.4(4.9-8.4) & $6.9(4.8-7.6)$ & $9.4(7.3-11.5)$ & 0.014 \\
\hline Neutrophils, $\times 10^{\wedge} 9 /$ per $L$ & $5.7(3.1-6.6)$ & $5.0(2.9-5.5)$ & $8.1(6.2-10.0)$ & 0.002 \\
\hline Lymphocytes, $\times 10^{\wedge} 9 /$ per $\mathrm{L}$ & I.I(0.7-I.5) & $1.3(0.8-1.6)$ & $0.7(0.5-0.9)$ & 0.002 \\
\hline Monocytes, $\times 10^{\wedge} 9 /$ per $L$ & $0.5(0.3-0.6)$ & $0.5(0.4-0.6)$ & $0.5(0.3-0.7)$ & 0.376 \\
\hline Eosinophils, $\times 10^{\wedge} 9 /$ per $\mathrm{L}$ & $0.1(0.1-0.1)$ & $0.1(0.0-0.1)$ & $0.0(0.0-0.0)$ & $<0.001$ \\
\hline Basophils, $\times 10^{\wedge} 9 /$ per $L$ & $0.0(0.0-0.0)$ & $0.0(0.0-0.0)$ & $0.0(0.0-0.0)$ & $0.28 \mathrm{I}$ \\
\hline Platelets, $\times 10^{\wedge} 9 /$ per $\mathrm{L}$ & $220.7(152.0-290.5)$ & $228.7(169.5-298.5)$ & $192.7(113.0-286.0)$ & 0.023 \\
\hline $\mathrm{T}$ cells $(\mathrm{CD} 3+\mathrm{CD}$ 19-) $/ \mu \mathrm{L}$ & $786.8(3 \mid 7.5-1070.0)$ & $1047.7(900.0-1196.0)$ & $357.2(179.5-490.5)$ & $<0.001$ \\
\hline $\mathrm{B}$ cells $(\mathrm{CD} 3-\mathrm{CD} / 9+) / \mu \mathrm{L}$ & I57.5(68.0-200.5) & I66. I (84.5-200.8) & | $43.4(33.0-225.0)$ & 0.569 \\
\hline Th cells $(\mathrm{CD} 3+\mathrm{CD} 4+) / \mu \mathrm{L}$ & $4977(200.0-691.0)$ & $655.3(497.0-837.0)$ & $238.1(107.5-288.5)$ & $<0.001$ \\
\hline Ts cells $(\mathrm{CD} 3+\mathrm{CD} 8+) / \mu \mathrm{L}$ & $259.9(89.5-346.5)$ & $352.2(265.0-388.0)$ & $107.9(55.5-150.0)$ & $<0.001$ \\
\hline NK cells (CD3-/CDI6+CD56+) / $\mu \mathrm{L}$ & $163.2(54.0-2 \mid 1.5)$ & $224.3(\mid 38.8-297.8)$ & $62.5(\mid 15.5-113.0)$ & $<0.001$ \\
\hline
\end{tabular}

Notes: *Serum cytokine levels were tested in 96 patients (74 cases recovered, 22 cases died), while lymphocyte subgroups were tested in 45 patients (28 recovered, 17 died). Abbreviations: IgM, immunoglobulin M; IgG, immunoglobulin G; IL, interleukin; TNF- $\alpha$, tumor necrosis factor $\alpha$; T cell, T lymphocyte; B cell, B lymphocyte; Th cell, helper T lymphocyte; Ts cell, suppressor T lymphocyte; NK cell, nature killer cell.

factors, and epidemiological history have been well studied, the distribution profile and role of SARS-Cov-2-specific immunoglobulins remain unclear. $^{12-14}$ Host immune response plays a key role in virus elimination. Previous reports of histological examinations of the lungs of patients who died due to COVID-19 have revealed bilateral diffuse 
Table 3 Clinical Characteristics of Patients in the Matched Cohort Study

\begin{tabular}{|c|c|c|c|c|}
\hline & Total $(n=69 *)$ & Recovered $(n=46 *)$ & Deceased $(n=23 *)$ & $P$ value \\
\hline Age, years & $68.8(61.5-77.5)$ & $68.2(61.0-75.8)$ & $70.1(65.0-79.0)$ & 0.465 \\
\hline Male & $45(65)$ & $28(61)$ & $17(74)$ & 0.284 \\
\hline Any comorbidities & $54(78)$ & $32(70)$ & $22(96)$ & 0.013 \\
\hline Positive SARS-CoV-2 RNA & $55(80)$ & $38(83)$ & $17(74)$ & 0.397 \\
\hline Hospital stay, days & $24.4(13.0-31.5)$ & $24.1(11.8-31.5)$ & $24.9(21.0-32.0)$ & 0.804 \\
\hline Hemoglobin, $g / L$ & $127.8(118.0-138.0)$ & $127.6(118.5-137.3)$ & $128.2(1 \mid 4.0-141.0)$ & 0.397 \\
\hline Alanine aminotransferase & $30.7(17.0-39.5)$ & $31.1(16.0-41.0)$ & $30.0(18.0-39.0)$ & 0.803 \\
\hline Aspartate aminotransferase & $34.1(21.0-42.5)$ & $32.0(20.8-39.3)$ & $38.3(24.0-53.0)$ & 0.165 \\
\hline Albumin, $g / L$ & $34.8(29.9-38.6)$ & $36.3(31.9-41.7)$ & $31.7(27.9-35.0)$ & 0.010 \\
\hline Total bilirubin, $\mu \mathrm{mol} / \mathrm{L}$ & $13.0(8.2-15.7)$ & $12.6(7.4-15.0)$ & $14.0(9.2-17.3)$ & 0.456 \\
\hline Lactate dehydrogenase, U/L & $354.1(215.5-458.5)$ & $321.7(212.8-354.8)$ & $419.0(273.0-524.0)$ & 0.082 \\
\hline Blood urea nitrogen, $\mathrm{mmol} / \mathrm{L}$ & $8.0(4.5-8.5)$ & $5.7(4.1-6.6)$ & $12.6(6.0-11.6)$ & $<0.001$ \\
\hline Creatinine, $\mu \mathrm{mol} / \mathrm{L}$ & $102.0(65.5-98.0)$ & $79.8(60.8-89.0)$ & $146.4(74.0-122.0)$ & 0.001 \\
\hline Prothrombin time, seconds & $14.7(13.6-15.2)$ & $13.9(13.1-14.6)$ & $16.5(14.6-16.6)$ & 0.010 \\
\hline D-dimer, $\mu g / m L$ & $4.1(0.4-3.7)$ & $2.8(0.3-2.3)$ & $6.9(0.8-17.7)$ & 0.006 \\
\hline $\operatorname{lgM}, \mathrm{AU} / \mathrm{mL}$ & |25.4(17.5-||9.8) & $72.3(13.0-56.2)$ & $231.6(30.4-296.2)$ & 0.006 \\
\hline $\lg \mathrm{G}, \mathrm{AU} / \mathrm{mL}$ & $213.6(142.7-217.8)$ & $203.0(136.5-247.2)$ & $234.8(157.3-295.5)$ & 0.223 \\
\hline Antibody detection after onset, days & $31.7(27.0-37.5)$ & $32.7(28.8-40.0)$ & $29.7(24.0-35.0)$ & 0.130 \\
\hline $\mathrm{IL} 2 \mathrm{R}, \mathrm{U} / \mathrm{mL}$ & $882.9(393.0-1326.0)$ & $708.8(358.0-1010.0)$ & 1223.2(580.0-1780.8) & 0.002 \\
\hline IL6, pg/mL & $98.5(4.2-64.9)$ & $37.0(2.9-40.5)$ & $213.5(18.8-136.4)$ & $<0.001$ \\
\hline $\mathrm{IL} 8, \mathrm{pg} / \mathrm{mL}$ & $55.1(10.8-43.1)$ & $33.8(10.3-34.1)$ & $96.8(13.3-84.0)$ & 0.044 \\
\hline ILI0, pg/mL & $5.2(0.0-7.5)$ & $3.6(0.0-6.0)$ & $8.3(0.0-11.4)$ & 0.070 \\
\hline TNF- $\alpha, p g / m L$ & $10.6(6.9-13.6)$ & $8.8(6.0-13.0)$ & $14.0(8.3-15.5)$ & 0.025 \\
\hline Leucocytes, $\times 10^{\wedge 9 / \text { per } \mathrm{L}}$ & $7.1(4.7-8.4)$ & $6.4(4.5-7.1)$ & $9.4(7.3-11.5)$ & 0.004 \\
\hline Neutrophils, $\times 10^{\wedge 9 / \text { per } \mathrm{L}}$ & $5.5(2.9-7.0)$ & $4.6(2.6-5.7)$ & $8.2(6.2-10.0)$ & $<0.001$ \\
\hline Lymphocytes, $\times 10^{\wedge} 9 /$ per $L$ & $1.0(0.6-1.4)$ & $1.1(0.7-1.5)$ & $0.7(0.5-0.9)$ & 0.017 \\
\hline Monocytes, $\times 10^{\wedge} 9 /$ per $\mathrm{L}$ & $0.5(0.4-0.6)$ & $0.5(0.5-0.6)$ & $0.5(0.3-0.7)$ & 0.848 \\
\hline Eosinophils, $\times 10^{\wedge 9 / \text { per } L}$ & $0.1(0.0-0.1)$ & $0.1(0.0-0.1)$ & $0.0(0.0-0.0)$ & 0.007 \\
\hline Basophils, $\times 10^{\wedge} 9 /$ per $\mathrm{L}$ & $0.0(0.0-0.0)$ & $0.0(0.0-0.0)$ & $0.0(0.0-0.0)$ & 0.968 \\
\hline Platelets, $\times 10^{\wedge} 9 /$ per $\mathrm{L}$ & $222.3(150.0-300.0)$ & $225.4(\mid 58.5-301.5)$ & $212.9(\mid 13.0-295.0)$ & 0.267 \\
\hline $\mathrm{T}$ cells (CD3+CDI9-) / $/ \mathrm{L}$ & $753.7(269.0-1071.0)$ & | 1 28.2(933.0-1302.8) & $357.2(179.5-490.5)$ & $<0.001$ \\
\hline$B$ cells $(C D 3-C D \mid 9+) / \mu \mathrm{L}$ & $158.7(53.0-200.0)$ & $173.2(72.0-200.3)$ & $143.4(33.0-225.0)$ & 0.531 \\
\hline Th cells $(\mathrm{CD} 3+\mathrm{CD} 4+) / \mu \mathrm{L}$ & $482.2(170.0-802.0)$ & $7 \mid 2.8(5 \mid 4.3-857.0)$ & $238.1(107.5-288.5)$ & $<0.001$ \\
\hline Ts cells $(\mathrm{CD} 3+\mathrm{CD} 8+) / \mu \mathrm{L}$ & $244.5(84.0-339.0)$ & $373.4(265.5-396.3)$ & $107.9(55.5-150.0)$ & $<0.001$ \\
\hline NK cells $(C D 3-/ C D 16+C D 56+) / \mu \mathrm{L}$ & $152.9(28.0-211.0)$ & $238.4(139.0-310.0)$ & $62.5(15.5-113.0)$ & $<0.001$ \\
\hline
\end{tabular}

Notes: *Serum cytokine levels were tested in 65 patients ( 43 cases recovered, 22 cases died), while lymphocyte subgroups were tested in 35 patients (I8 recovered, 17 died).

Abbreviations: IgM, immunoglobulin M; IgG, immunoglobulin G; IL, interleukin; TNF- $\alpha$, tumor necrosis factor $\alpha$; T cell, T lymphocyte; B cell, B lymphocyte; Th cell, helper T lymphocyte; Ts cell, suppressor T lymphocyte; NK cell, nature killer cell. 


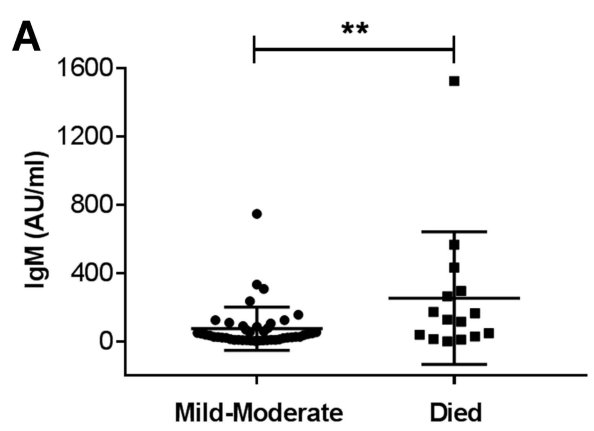

D

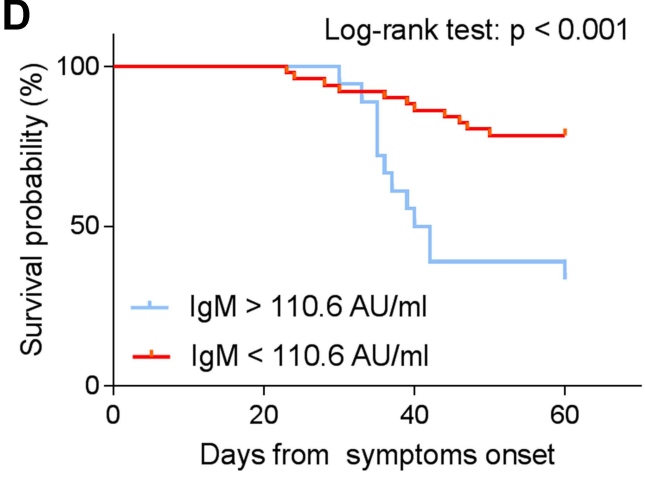

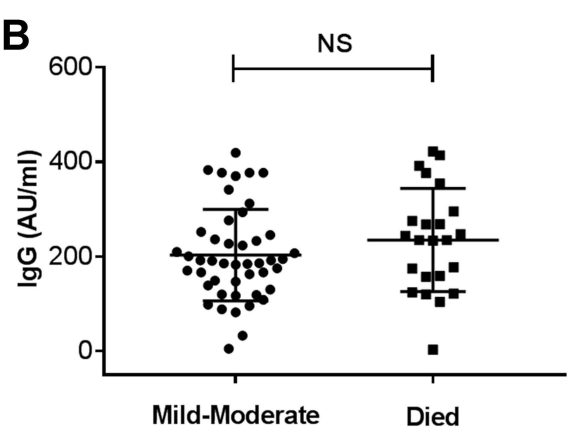

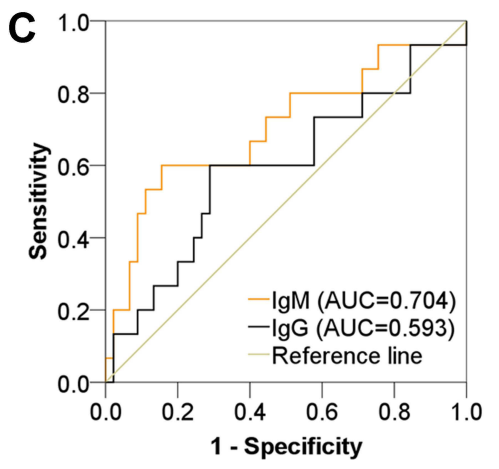

E

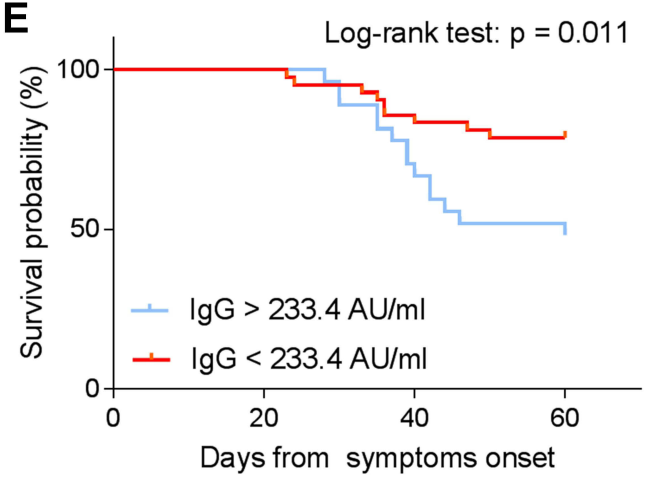

Figure 3 Serum SARS-Cov-2-specific IgM and IgG antibodies in the age-matched cohort study. (A) Serum IgM levels were significantly higher in the deceased group in the cohort study. **P < 0 0I. (B) No difference was found in the lgG levels between the recovered group and the deceased group. (C) Accuracy of serum IgM levels and IgG levels in predicting the clinical outcomes of COVID-19 patients. (D) Kaplan-Meier estimates for survival based on IgM (stratified according to best cut-off). (E) Kaplan-Meier estimates for survival based on IgG (stratified according to best cut-off).

Abbreviation: NS, non-significant.

alveolar damage with cellular exudates, accompanied by interstitial mononuclear inflammatory infiltrates. ${ }^{15}$ Peripheral T cell counts have been shown to be substantially reduced, although the present $\mathrm{T}$ cells were hyperactivated. ${ }^{15,16}$ Elevated levels of SARS-CoV-2-specific antibodies have been previously detected in COVID-19 patients. ${ }^{8}$ SARS-CoV-1-specific IgG antibodies persist for a relatively longer time (more than three months), although SARS-CoV-1-specific IgM peaks in the third week and remains measurable for a much shorter period. ${ }^{11}$ Similarly, SARS-CoV-2-specific IgM levels peaked in the fourth week after the onset of COVID-19 and then decreased more slowly than SARS-CoV-1-specific IgM; in contrast, serum IgG levels rose quickly in the first two weeks and remained high for up to over 8 weeks. The relatively slow development but longer persistence of the immune response has been attributed to a relatively weaker pathogenicity and prolonged viral shedding. ${ }^{6,13}$

In our study, both the matched and unmatched analyses yielded similar results: serum IgM levels were higher in the non-survivors, serum IgM levels were negatively correlated with the clinical outcomes in the correlation analysis, and the IgM levels were predictive of the clinical outcomes assessed with ROC curve analysis. Moreover, as shown by Kaplan-Meier survival curves, COVID-19 patients with high IgM or IgG (stratified according to the best cut-off) exhibited significantly lower overall survival. Therefore, we infer that elevated SARS-CoV-2-specific IgM levels indicate a poor outcome in patients with COVID-19. Similarly, another report indicated that critical patients showed significantly higher total antibody levels than noncritical cases at approximately 2 weeks after onset $(\mathrm{P}=0.02)$, but this association was not significant for either the IgM or IgG tests. ${ }^{17}$ Possible reasons for these observations are as follows: first, IL-6, which plays a key role in the development of cytokine storm, efficiently augments antibody production; ${ }^{18}$ second, the IgM level was positively correlated with the duration of viral RNA presence; older age correlated with a higher viral load, ${ }^{9}$ thereby indicating that high immunoglobulin levels are associated with high viral loads, and is a prognostic factor for poor outcomes. ${ }^{19}$ Although SARS-CoV-2-specific antibodies are associated with outcomes in COVID-19 patients, their role in virus elimination is not clear. For example, neutralizing antibodies bind to the surface spike protein of coronaviruses and mediate viral entry into IgG Fc receptor-expressing cells, thereby actively participating in 
viral elimination. ${ }^{20}$ Further studies are needed to ascertain the role of antibodies in the response to SARS-CoV-2 infection.

This study has some limitations. First, it is a singlecenter retrospective study with a limited number of cases. Second, since the majority of patients admitted to our hospital were critically ill, a population bias cannot be excluded from our study. Third, the viral load results were not available for our study group, because a qualitative method for the screening has been used.

\section{Conclusion}

Collectively, our study shows that elevated serum IgM levels are indicative of clinically unfavorable outcomes in patients with COVID-19, and provides insight into the interaction between immunoglobulin and host systems. A better understanding of SARS-CoV-2-specific IgGs is imperative to enhance the diagnostics during the COVID-19 pandemic, our study outcomes might be helpful in designing future diagnostic assays and epidemiologic surveys.

\section{Abbreviations}

AUC, area under the ROC curve; BUN, Blood urea nitrogen; CHD, Coronary heart disease; CKD, Chronic kidney disease; COPD, Chronic obstructive pulmonary disease; COVID-19, coronavirus disease 2019; IgM, Immunoglobulin $\mathrm{M}$; IgG, Immunoglobulin G; IL6, Interleukin 6; IQR, interquartile range; SARS-CoV-2, severe acute respiratory syndrome coronavirus 2 .

\section{Data Sharing Statement}

The raw data supporting the conclusions of this article will be made available by the author Jingjing $\mathrm{Li}$, without undue reservation.

\section{Compliance with Ethical Standards}

All procedures performed in studies involving human participants were in accordance with the ethical standards of the Lifespan institutional research committee and with the 1964 Helsinki declaration and its later amendments or comparable ethical standards.

\section{Acknowledgments}

This work was supported by the National Natural Science Foundation of China (No. 81702989) and Hubei Provincial Natural Science Foundation (No. 2020CFB765). We acknowledge all patients involved in the study and all health-care workers against COVID-19.

\section{Disclosure}

The authors declare that the research was conducted in the absence of any commercial or financial relationships that could be construed as a potential conflict of interest.

\section{References}

1. Chen N, Zhou M, Dong X, et al. Epidemiological and clinical characteristics of 99 cases of 2019 novel coronavirus pneumonia in Wuhan, China: a descriptive study. Lancet. 2020;395 (10223):507-513. doi:10.1016/S0140-6736(20)30211-7

2. Zhu N, Zhang D, Wang W, et al. A novel coronavirus from patients with pneumonia in China, 2019. $N$ Engl $J$ Med. 2020;382 (8):727-733. doi:10.1056/NEJMoa2001017

3. World Health Organization. Novel coronavirus (COVID-19) situation. Available from: https:/experience.arcgis.com/experience/ 685d0ace521648f8a5beeeee1b9125cd. Accessed December 21, 2021.

4. Li G, De Clercq E. Therapeutic options for the 2019 novel coronavirus (2019-nCoV). Nat Rev Drug Discov. 2020;19(3):149-150. doi:10.1038/d41573-020-00016-0

5. Dibo M, Battocchio EC, Dos Santos Souza LM, et al. Antibody therapy for the control of viral diseases: an update. Curr Pharm Biotechnol. 2019;20(13):1108-1121. doi:10.2174/13892010206 66190809112704

6. Channappanavar R, Perlman S. Pathogenic human coronavirus infections: causes and consequences of cytokine storm and immunopathology. Semin Immunopathol. 2017;39(5):529-539. doi:10.1007/s00281-017-0629-x

7. Gronvall G, Connell N, Kobokovich A, et al. Developing a National strategy for serology (antibody testing) in the United States. 2020; Available from: https://www.centerforhealthsecurity.org/our-work /publications/developing-a-national-strategy-for-serology-antibodytesting-in-the-US. Accessed December 21, 2021.

8. Li Z, Yi Y, Luo X, et al. Development and clinical application of a rapid IgM-IgG combined antibody test for SARS-CoV-2 infection diagnosis. J Med Virol. 2020. doi:10.1002/jmv.25727

9. To KK-W, Tsang OT-Y, Leung W-S, et al. Temporal profiles of viral load in posterior oropharyngeal saliva samples and serum antibody responses during infection by SARS-CoV-2: an observational cohort study. Lancet Infect Dis. 2020;20(5):565-574. doi:10.1016/S14733099(20)30196-1

10. Thevarajan I, Nguyen THO, Koutsakos M, et al. Breadth of concomitant immune responses prior to patient recovery: a case report of non-severe COVID-19. Nat Med. 2020;26(4):453-455. doi:10.1038/ s41591-020-0819-2

11. $\mathrm{Li}$ G, Chen X, Xu A. Profile of specific antibodies to the SARS-associated coronavirus. $N$ Engl J Med. 2003;349(5):508-509. doi:10.1056/NEJM200307313490520

12. Kucharski AJ, Russell TW, Diamond C, et al. Early dynamics of transmission and control of COVID-19: a mathematical modelling study. Lancet Infect Dis. 2020;20(5):553-558. doi:10.1016/S14733099(20)30144-4

13. Zhou F, Yu T, Du R, et al. Clinical course and risk factors for mortality of adult inpatients with COVID-19 in Wuhan, China: a retrospective cohort study. Lancet. 2020;395(10229):1054-1062. doi:10.1016/S0140-6736(20)30566-3

14. Huang C, Wang Y, Li X, et al. Clinical features of patients infected with 2019 novel coronavirus in Wuhan, China Lancet. 2020;395 (10223):497-506. doi:10.1016/S0140-6736(20)30183-5

15. Xu Z, Shi L, Wang Y, et al. Pathological findings of COVID-19 associated with acute respiratory distress syndrome. Lancet Respir Med. 2020;8(4):420-422. doi:10.1016/S2213-2600(20) 30076-X 
16. Qin C, Zhou L, Hu Z, et al. Dysregulation of immune response in patients with COVID-19 in Wuhan, China. Clin Infect Dis. 2020;71 (15):762-768. doi:10.1093/cid/ciaa248

17. Zhao J, Yuan Q, Wang H, et al. Antibody responses to SARS-CoV-2 in patients of novel coronavirus disease 2019. Clin Infect Dis. 2020;71(16):2027-2034. doi:10.1093/cid/ciaa344

18. Nawata Y, Stall AM, Herzenberg LA, et al. Surface immunoglobulin ligands and cytokines differentially affect proliferation and antibody production by human CD5+ and CD5-B lymphocytes. Int Immunol. 1990;2(7):603-614. doi:10.1093/intimm/2.7.603
19. Weilie chen W, Lan Y, Yuan X, et al. Detectable 2019-nCoV viral RNA in blood is a strong indicator for the further clinical severity. Emerg Microbes Infect. 2020;9(1):469-473. doi:10.1080/ 22221751.2020.1732837

20. Wan Y, Shang J, Sun S, et al. Molecular mechanism for antibody-dependent enhancement of coronavirus entry. $J$ Virol. 2020;94(5). doi:10.1128/JVI.02015-19

\section{Publish your work in this journal}

The International Journal of General Medicine is an international, peer-reviewed open-access journal that focuses on general and internal medicine, pathogenesis, epidemiology, diagnosis, monitoring and treatment protocols. The journal is characterized by the rapid reporting of reviews, original research and clinical studies across all disease areas. The manuscript management system is completely online and includes a very quick and fair peer-review system, which is all easy to use. Visit http://www.dovepress.com/ testimonials.php to read real quotes from published authors. 